\title{
A class of corners of a Leavitt path algebra
}

\author{
Trinh Thanh Deo
}

\begin{abstract}
Tóm tắt- Let $E$ be a directed graph, $K$ a field and $L_{K}(E)$ the Leavitt path algebra of $E$ over $K$. The goal of this paper is to describe the structure of a class of corners of Leavitt path algebras $L_{K}(E)$. The motivation of this work comes from the paper "Corners of Graph Algebras" of Tyrone Crisp in which such corners of graph $C^{*}$-algebras were investigated completely. Using the same ideas of Tyrone Crisp, we will show that for any finite subset $X$ of vertices in a directed graph $E$ such that the hereditary subset $H_{E}(X)$ generated by $X$ is finite, the corner $\left(\sum_{v \in X} v\right) L_{K}(E)\left(\sum_{v \in X} v\right)$ is isomorphic to the
\end{abstract} Leavitt path algebra $L_{K}\left(E_{X}\right)$ of some graph $E_{X}$. We also provide a way how to construct this graph $E_{X}$.

Tù khóa- Leavitt path algebra, graph, corner.

\section{INTRODUCTION}

$\mathrm{L}$ eavitt path algebras for graphs were developed independently by two groups of mathematicians. The first group, which consists of Ara, Goodearl and Pardo, was motivated by the $K$-theory of graph algebras. They introduced Leavitt path algebras [3] in order to answer analogous $K$-theoretic questions about the algebraic Cuntz-Krieger algebras. On the other hand, Abrams and Aranda Pino introduced Leavitt path algebras $L_{K}(E)$ in [2] to generalise Leavitt's algebras, specifically the algebras $L_{K}(1, n)$.

The goal of this paper is to describe the structure of a class of corners of Leavitt path algebras $L_{K}(E)$. The motivation of this work comes from [4] in which such corners of graph $C^{*}$-algebras were investigated completely. Using the same ideas from [4], we will show that for any finite subset $X$ of vertices in a directed graph $E$ such that the hereditary subset $H_{E}(X)$ generated

Ngày nhận bản thảo: 03-01-2017; Ngày chấp nhận đăng: 07-03-2018; Ngày đăng: 15-10-2018.

Author Trinh Thanh Deo - University of Science, VNUHCM (email: ttdeo@hcmus.edu.vn) by $X$ is finite, the corner $\left(\sum_{v \in X} v\right) L_{K}(E)\left(\sum_{v \in X} v\right)$ is isomorphic to the Leavitt path algebra $L_{K}\left(E_{X}\right)$ of some graph $E_{X}$. We also provide a way how to construct this graph $E_{X}$.

The graph $C^{*}$-algebra of an arbitrary directed graph $E$ plays an important role in the theory of $C^{*}$-algebras. In 2005, G. Abrams and G. ArandaPino [2] defined the algebra $L_{K}(E)$ of a directed graph $E$ over a field $K$ which was the universal $K$ algebra, named Leavitt path algebra, generated by elements satisfying relations similar to the ones of the generators in the graph $C^{*}$-algebra of $E$ and was considered as a generalization of Leavitt algebras $L(1, n)$. Historically, G. Abrams and G. Aranda-Pino found his inspiration from results on graph $C^{*}$-algebras to define Leavitt path algebras, so that one of first topics in Leavitt path algebras was to find some analogues for Leavitt path algebras of graph $C^{*}$-algebras such as in [1, 5]. In [4], the class of corners $P_{X} C^{*}(E) P_{X}$ were investigated completely when $X$ was a finite subset of $E^{0}$ with $H_{E}(X)$ was finite. In the present note, we consider the similar problem for Leavitt path algebra $L_{K}(E)$. In the next section, we recall briefly the notation and results on the graph theory. In Section 3, we present the way to find a graph $E_{X}$ and an isomorphism of $\left(\sum_{v \in X} v\right) L_{K}(E)\left(\sum_{v \in X} v\right)$ and $L_{K}\left(E_{X}\right)$. The ideas and arguments we use in Section 3 is almost similar to [4] but there are two important things here: arguments in [4] will be rewritten according to the language of Leavitt path algebras and, secondary, we will modify a little bit these arguments to pass difficulties of hypothesis between graph $C^{*}$ algebras and Leavitt path algebras.

\section{PRELIMINARIES ON GRAPH THEORY}

A directed graph $E=\left(E^{0}, E^{1}, r, s\right)$ consists of two countable sets $E^{0}, E^{1}$ and maps $r, s: E^{0} \rightarrow E^{1}$. 
The elements of $E^{0}$ are called vertices and the elements of $E^{1}$ edges. For each edge $e, s(e)$ is the source of $e, r(e)$ is the range of $e$, and $e$ is said to be an edge from s(e) to $r(e)$. A graph is row-finite if $s^{-1}(v)$ is a finite set for every $v \in E^{0}$. If $E^{0}$ and $E^{1}$ are finite, then we say that $E$ is finite. A vertex which emits no edges is a sink. A path $\mu$ in the graph $E$ is a sequence of edges $\mu=e_{1} \ldots e_{n}$ such that $r\left(e_{i}\right)=s\left(e_{i+1}\right)$ for $i=1, \ldots, n-1$. We call $s\left(e_{1}\right)$ the source of $\mu$, denote by $s(\mu) ; r\left(e_{1}\right)$ is the range of $\mu$, denote by $r(\mu)$; the number $n$ is the length of $\mu$. If $\mu$ and $\nu$ are paths such that $\mu=v \eta$ for some path $\eta$, then we say that $v$ is an initial subpath of $\mu$, denote by $\nu \prec \mu$.

For $n \geq 2$, let $E^{n}$ be the set of paths of length $n$, and denote by $E^{*}=\bigcup_{n \geq 0} E^{n}$. If we consider every vertex as a path of length 0 and edge as a path of length 1 , then $E^{*}$ is the set of paths of length $n \geq 0$.

Let $F$ be a subgraph of $E$, that is, $F$ is a graph whose vertices and edges form subsets of the vertices and edges of $E$ respectively. For vertices $u, v \in E^{0}$ we write $u \geq_{F} v$ if there is a path $\mu \in F^{*}$ such that $s(\mu)=u$ and $r(\mu)=v$. We say that a subset $X \subseteq E^{0}$ is hereditary if $v \in X$ and $u \in E^{0}$ such that $v \geq_{F} u$, then $u \in X$. For any subset $Y \subseteq E^{0}$ we shall denote by $H_{E}(Y)$ the smallest hereditary subset of $E^{0}$ containing $Y$. The set $H_{E}(Y) \backslash Y$ is referred to as the hereditary complement of $Y$ in $E$. The subgraph $T=\left(T^{0}, T^{1}, r, s\right)$ is called a directed forest in $E$ if it satisfies the two following conditions:

(1) $\mathrm{T}$ is acyclic, that is, for every path $e_{1} \ldots e_{n}$ in $T$, one has $r\left(e_{i}\right) \neq s\left(e_{j}\right)$ if $i \geq j$.

(2) For each vertex $v$ in $T^{0},\left|T^{1} \cap r^{-1}(v)\right| \leq 1$.

If $T$ is a directed forest of $E$, then $T^{r}$ denotes the subset of $T^{0}$ consisting of those vertices $v$ with $\left|T^{1} \cap r^{-1}(v)\right|=0$, and $T^{l}$ denotes the subset of $T^{0}$ consisting of those vertices $v$ with $\left|T^{1} \cap s^{-1}(v)\right|=0$. The sets $T^{r}$ and $T^{l}$ are called the roots and the the leaves of $T$.

The following lemmas are from [4].

Lemma 1 ([4, Lemma 2.2]). Let $T$ be a row-finite, path-finite directed forest in a directed graph $E$. Then the following statements hold: i) For each $v \in T^{0}$ there exists a unique path $\tau_{v}$ in $T^{*}$ with source in $T^{r}$ and range $v$. Moreover, for $u, v \in T^{0}, v \geq_{T} u \Leftrightarrow \tau_{v} \prec \tau_{u} \Leftrightarrow$ there exists a unique path $\tau_{v, u} \in T^{*}$ with source $v$ and range $u$.

ii) For each $v \in T^{0}$ there exist at most finitely many vertices $u \in T^{0}$ with $v \geq_{T} u$.

iii) For each $v \in T^{0}$ there exists at least one $u \in T^{l}$ such that $v \geq_{T} u$.

iv) Suppose $u, v \in T^{0}$ have $\tau_{v} \prec \tau_{u}$ and $u \neq v$. Then there exists a unique edge $e \in s^{-1}(v) \cap T^{1}$ such that $\tau_{v} e^{\prec} \tau_{u}$. If $f \in s^{-1}(v) \cap T^{1}$ satisfies $\tau_{u} \prec \tau_{v} f$, then $f=e$ and $\tau_{v} e=\tau_{u}$.

The key result of building a new graph $E_{X}$ in this paper is the existence of the directed forest with given roots. In general, a forest with given roots [4, Lemma 3.6] may not exists, but in some special cases, we can find such forest.

Lemma 2. Let $E=\left(E^{0}, E^{1}, r, s\right)$ be a directed graph and $X$ a finite subset of $E^{0}$. If $H_{E}(X)$ is finite, then there is a row-finite, finite-path directed forest $T$ in $E$ with $T^{r}=X$ and $T^{0}=H_{E}(X)$.

Proof. This lemma is just a corollary of $[4$, Lemma 3.6].

\section{RESULTS}

We have mentioned graph $C^{*}$-algebras in the Introduction, but this paper focus only on Leavitt path algebras. In this section, before going to the main goal of paper, we briefly recall just the definition of the Leavitt path algebra of a graph. For a definition of these algebras with remarks one can see in [2].

Given a graph $E=\left(E^{0}, E^{1}, r, s\right)$, we denote the new set of edges $\left(E^{1}\right)^{*}$, which is a copy of $E^{1}$ but with the direction of each edge reversed; that is, if $e \in E^{1}$ runs from $u$ to $v$, then $e^{*} \in\left(E^{1}\right)^{*}$ runs from $v$ to $u$. We refer to $E^{1}$ as the set of real edges and $\left(E^{1}\right)^{*}$ as the set of ghost edges.

The path $p=e_{1} \ldots e_{n}$ made up of only real edges is called the real path, and we denote the ghost path $e_{n}{ }^{*} \ldots e_{1}{ }^{*}$ by $p^{*}$.

Let $K$ be a field and $E$ a directed graph. The Leavitt path $K$-algebra $L_{K}(E)$ of $E$ over $K$ is the (universal) $K$-algebra generated by a set $\left\{v \mid v \in E^{0}\right\}$ of pairwise orthogonal idempotents, together with 
a set of variables $\left\{e, e^{*} \mid e \in E^{1}\right\}$ which satisfy the following relations:

(1) $s(e) e=e r(e)=e$ for all $e \in E^{1}$.

(2) $\quad r(e) e^{*}=e^{*} s(e)=e$ for all $e \in E^{1}$.

(3) $e^{*} e^{\prime}=\delta_{e, e^{\prime}} r(e)$ for all $e, e^{\prime} \in E^{1}$.

(4) $v=\sum_{e \in s^{-1}(v)} e e^{*}$ for every $v \in E^{0}$ that emits edges.

Let $T$ be a path-finite directed forest in $E$. For each $v \in T^{0}$ let $\tau_{v} \in T^{*}$ be the path given by part (i) of Lemma 1 (in particular, for $v \in X, \tau_{v}=v$ ). Now for each $v \in T^{0}$, define

$$
Q_{v}:=\tau_{v} \cdot \tau_{v}^{*}-\sum_{e \in T^{1} \cap s^{-1}(v)} \tau_{v} \cdot e e^{*} \cdot \tau_{v}^{*} .
$$

Clearly, $Q_{v}^{*}=Q_{v}$.

Lemma 3. For each $v \in T^{0}, Q_{v}=0$ if and only if $\varnothing \neq s^{-1}(v) \subseteq T^{1}$. Also,

$$
\tau_{v} \cdot \tau_{v}^{*}=\sum_{u \in T^{0}, v \geq_{T} u} Q_{u} .
$$

Proof. The proof of this lemma is just a slight modification of [4, Lemma 3.7]. We first show the first statement. The fact that if $\varnothing \neq s^{-1}(v) \subseteq T^{1}$, then $Q_{v}=0$ is from first arguments in [4, Lemma 3.7]. Now we show that if $Q_{v}=0$, then $\varnothing \neq s^{-1}(v) \subseteq T^{1}$ for every $v \in T^{0}$. If $v$ is a sink in $E$ then $Q_{v}=\tau_{v} \tau_{v}^{*} \neq 0$. If $v$ emits an edge $f \in E^{1} \backslash T^{1}$ then

$$
\begin{gathered}
Q_{v}=\tau_{v} \tau_{v}^{*}-\sum_{e \in T^{1} \cap s^{-1}(v)} \tau_{v} \cdot e e^{*} \cdot \tau_{v}^{*} \\
=\tau_{v} \cdot f f^{*} \cdot \tau_{v}^{*}+\tau_{v}\left(\sum_{e \in s^{-1}(v) \backslash\left(T^{1} \cup\{f\}\right)} e e^{*}\right) \tau_{v}^{*} \neq 0 .
\end{gathered}
$$

The rest of the proof is from the second part of [4, Lemma 3.7] with replacing $S_{\tau(v)}$ and $S_{\tau(v)}^{*}$ by $\tau(v)$ and $\tau(v)^{*}$ respectively for every $v \in T^{0}$.

Let $E$ be a directed graph, and assume that $X$ is a finite subset of $E^{0}$ such that $H_{E}(X)$ is finite. By Lemma 2, there exists a row-finite, path-finite directed forest $T$ in $E$ with $T^{r}=X$ and $T^{0}=H_{E}(X)$.
Let

$$
V(T):=T^{0} \backslash\left\{v \in T^{0}: \varnothing \neq s^{-1}(v) \subseteq T^{1}\right\},
$$

that is, $V(T)$ consists of vertices which are sinks and emit at least one edge not belonging to $T$. By Lemma 3, $Q_{v} \neq 0$ iff $v \in V(T)$.

For each $e$ in $E^{1} \backslash T^{1}$ and $u \in V(T)$ such that

$$
s(e), r(e) \in T^{0}, r(e) \geq_{T} u,
$$

we define $p_{e, u}$ as the path $e \tau_{r(e), u}$. Using the same techniques as in the proof of [4, Lemma 3.9], we obtain that each edge $e$ in $E^{1} \backslash T^{1}$ with $s(e) \in T^{0}$ gives at least one path $p_{e, u}$ for some $u \in V(T)$ such that $r(e) \geq_{T} u$. In particular, if $v \in T^{0}$ is a singular vertex of $E$ then the set of all $p_{e, u}$ with source $v$ is finite.

For $p_{e, u}$ with $u \in V(T)$ and $r(e) \geq_{T} u$, define

$$
T_{e, u}:=\tau_{s(e)} \cdot e \cdot \tau_{r(e)}^{*} \cdot Q_{u} \text {. }
$$

We have:

Proposition 4. For each $u, v \in V(T)$, we have:
i) $Q_{v} Q_{w} \neq 0$ iff $v=w$.
ii) $\quad T_{e, u}^{*} T_{e, u}=Q_{u}$ and $T_{e, u}^{*} T_{f, v}=Q_{u} Q_{v}$.
iii) $\quad T_{e, u} T_{e, u}^{*} Q_{s(e)}=T_{e, u} T_{e, u}^{*}$.

Proof. Suppose $v$ and $w$ are distinct elements of $V(T)$ such that $Q_{v} Q_{w} \neq 0$, then $\tau_{v}^{*} \tau_{w} \neq 0$. It is easy to see that one of $\tau_{v}$ and $\tau_{w}$ is an initial subpath of the other. Assume, without loss of generality, that $\tau_{w} \prec \tau_{v}$, and let $f \in T^{1} \cap s^{-1}(w)$ be the edge given by Lemma 1 (iv). Then

$$
\sum_{e \in T^{1} \cap s^{-1}(w)} \tau_{v}^{*} \tau_{w} \cdot e e^{*} \cdot \tau_{w}^{*}=\tau_{v}^{*} \tau_{w} \cdot f f^{*} \cdot \tau_{w}^{*},
$$

because $f$ is a unique edge in $T^{1} \cap s^{-1}(w)$ with the property that $\tau_{w} f \prec \tau_{v}$. Now

$$
\tau_{v}^{*} \tau_{w} . f f^{*} \cdot \tau_{w}^{*}=\tau_{v}^{*}
$$

and thus

$$
\begin{gathered}
Q_{v} Q_{w}=Q_{v} \cdot \tau_{v} \tau_{v}^{*}\left(\tau_{w} \tau_{w}^{*}-\sum_{e \in T^{1} \cap s^{-1}(w)} \tau_{w} \cdot e e^{*} \cdot \tau_{w}^{*}\right) \\
=Q_{v}\left(\tau_{v} \tau_{v}^{*}-\tau_{v} \tau_{v}^{*}\right)=0 .
\end{gathered}
$$

Hence $Q_{v} Q_{w} \neq 0$ if and only if $v=w$ 
ii) Turning our attention to the $T_{e, u}$, fix $p_{e, u}$ with $u \in V(T)$ and $r(e) \geq_{T} u$. By definition of $p_{e, u}$ we must have $\tau_{r(e)} \prec \tau_{u}$. Therefore

$$
\begin{aligned}
T_{e, u}^{*} T_{e, u} & =Q_{u} \cdot \tau_{r(e)}\left(e^{*} \tau_{s(e)}^{*} \cdot \tau_{s(e)} e\right) \tau_{r(e)}^{*} Q_{u} \\
& =Q_{u} \cdot \tau_{r(e)} \cdot r(e) \cdot \tau_{r(e)}^{*} \cdot Q_{u} \\
& =Q_{u} \cdot \tau_{r(e)} \tau_{r(e)}^{*} \cdot Q_{u}=Q_{u} .
\end{aligned}
$$

Take $p_{e, u}$ and $p_{f, v}$ with $u, v \in V(T)$ and suppose $T_{e, u}^{*} T_{f, v} \neq 0$. Now

$$
T_{e, u} T_{f, v}=Q_{u} \cdot \tau_{r(e)} \cdot e^{*} \tau_{s(e)}^{*} \cdot \tau_{s(f)} f \cdot \tau_{r(f)}^{*} \cdot Q_{v},
$$

and in order for this product to be nonzero we must have either

$$
\tau_{s(f)} f \prec \tau_{s(e)} e \text { or } \tau_{s(e)} e \prec \tau_{s(f)} f .
$$

Since neither $e$ nor $f$ belongs to $T^{1}$ (so that neither $e$ nor $f$ may be a part of any $\tau_{w}$ ), this implies that $\tau_{s(e)} e=\tau_{s(f)} f$, and so $e=f$. Putting $e=f$ in (2) gives

$$
T_{e, u}^{*} T_{f, v}=Q_{u} \cdot \tau_{r(e)} \cdot \tau_{r(e)}^{*} \cdot Q_{v}=Q_{u} Q_{v},
$$

and in order for this product to be nonzero we must have $u=v$.

iii) We have

$$
\begin{aligned}
&\left(\tau_{s(e)} e\right)^{*} Q_{s(e)}=\left(\tau_{s(e)} e\right)^{*}\left(\tau_{s(e)} \tau_{s(e)}^{*}-\right. \\
&\left.\sum_{f \in T^{1} \cap s^{-1}(s(e))}\left(\tau_{s(e)} f\right)\left(\tau_{s(e)} f\right)^{*}\right) \\
&=\left(\tau_{s(e)} e\right)^{*}\left(\tau_{s(e)} e\right)\left(\tau_{s(e)} e\right)^{*}-0=\left(\tau_{s(e)} e\right)^{*} .
\end{aligned}
$$

Since $e \notin T^{1}, \tau_{s(e)} e$ is not an initial subpath of any $\tau_{s(e)} f$ for $f \in T^{1}$. Thus

$$
\begin{aligned}
T_{e, u} T_{e, u}^{*} Q_{s(e)} & \left.=T_{e, u} Q_{v} \cdot \tau_{r(e)} \cdot\left(\tau_{s(e)} e\right)^{*} \cdot Q_{s(e)}\right) \\
& =T_{e, u} Q_{v} \cdot \tau_{r(e)}\left(\tau_{s(e)} e\right)^{*}=T_{e, u} T_{e, u}^{*} .
\end{aligned}
$$

Proposition 5. For each $u, v \in V(T)$, we have:

i) $\quad Q_{u} Q_{v}=\delta_{u v} Q_{u}$.

ii) $T_{e, u} Q_{u}=T_{e, u}=Q_{s(e)} T_{e, u}$; and

$Q_{u} T_{e, u}^{*}=T_{e, u}^{*}=T_{e, u}^{*} Q_{s(e)}$.

iii) $T_{e, u}^{*} T_{f, v}=\delta_{u v} Q_{u}$.

iv) For each $v \in V(T)$, we have

$Q_{v}=\sum_{s(e)=v} T_{e, u} T_{e, u}^{*}$.
Proof. i) By Proposition 4i) and ii).

ii) By i) and by the definition of $T_{e, u}$ we have the first equation.

For the second equation, by Proposition 4iii), we have

$$
T_{e, u} T_{e, u}^{*} Q_{s(e)}=T_{e, u} T_{e, u}^{*} .
$$

It follows that

$$
Q_{s(e)} T_{e, u} T_{e, u}^{*}=T_{e, u} T_{e, u}^{*} .
$$

Therefore

$$
\begin{aligned}
Q_{s(e)} T_{e, u} & =Q_{s(e)} T_{e, u} T_{e, u}^{*} T_{e, u} \\
& =T_{e, u} T_{e, u}^{*} T_{e, u} \\
& =T_{e, u} .
\end{aligned}
$$

iii) By Proposition 4i) and ii).

iv) Suppose $v \in V(T)$ is nonsingular in $E$. Then, (CK2) in $L_{K}(E)$ gives

$$
v=\sum_{e \in s^{-1}(v)} e e^{*} .
$$

Now

$$
\begin{aligned}
Q_{v} & =\tau_{v} \tau_{v}^{*}-\sum_{e \in T^{1} \cap s^{-1}(v)}\left(\tau_{v} e\right)\left(\tau_{v} e\right)^{*} \\
& =\tau_{v} \cdot v \cdot \tau_{v}^{*}-\tau_{v}\left(\sum_{e \in T^{1} \cap s^{-1}(v)} e e^{*}\right) \tau_{v}^{*} \\
& =\tau_{v}\left(v-\sum_{e \in T^{1} \cap s^{-1}(v)} e e^{*}\right) \tau_{v}^{*} \\
& =\sum_{e \in s^{-1}(v) \backslash T^{1}} \tau_{v} e\left(\tau_{v} e\right)^{*} .
\end{aligned}
$$

Fix an edge $e \in s^{-1}(v) \backslash T^{1}$. This edge gives one path $p_{e, u}$ with source $v$ for each vertex $u \in V(T)$ with $r(e) \geq_{T} u$. The formula (1) of Lemma 3 gives

$$
\begin{aligned}
\left(\tau_{v} e\right)\left(\tau_{v} e\right)^{*}=\left(\tau_{v} e\right) r(e)^{3}\left(\tau_{v} e\right)^{*} & \\
= & \left(\tau_{v} e\right)\left(\tau_{r(e)}\right)^{*}\left(\tau_{r(e)} \tau_{r(e)}^{*}\right)^{2} \tau_{r(e)}\left(\tau_{v} e\right)^{*} \\
= & \left(\tau_{v} e\right) \tau_{r(e)}^{*}\left(\tau_{r(e)} \tau_{r(e)}^{*}\right)\left(\tau_{v} e \tau_{r(e)}^{*}\left(\tau_{r(e)} \tau_{r(e)}^{*}\right)\right)^{*} \\
& =\left(\tau_{v} e \tau_{r(e)}^{*}\left(\sum_{u \in T^{0}, r(e) \geq_{r} u} Q_{u}\right)\right)\left(\tau_{v} e \tau_{r(e)}^{*}\left(\sum_{u \in T^{0}, r(e) \geq_{r} u} Q_{u}\right)\right)^{*} \\
& =\left(\sum_{u \in V(T), r(e) \geq_{r} u} T_{e, u}\right)\left(\sum_{u \in V(T), r(e) \geq_{r} u} T_{e, u}\right)^{*} .
\end{aligned}
$$

Since for $u \neq u^{\prime}$ we have $T_{e, u} T_{e_{u^{\prime}}}^{*}=0$, this product expands as

$$
\left(\tau_{v} e\right)\left(\tau_{v} e\right)^{*}=\sum_{u \in V(T), r(e) \geq_{T} u} T_{e, u} T_{e, u}^{*} .
$$

Substituting (4) into (3) gives the Cuntz-Krieger identity 


$$
Q_{v}=\sum_{s(e)=v} T_{e, u} T_{e, u}^{*}
$$

and this final identity completes the proof of the proposition.

In view of Proposition 5, we can define the new graph $E_{X}$ as follows:

Definition 6. Let $E$ be a directed graph, and assume that $X$ is a finite subset of $E^{0}$ such that $H_{E}(X)$ is finite, and let $T$ be a row-finite, pathfinite directed forest in $E$ with $T^{r}=X$ and $T^{0}=H_{E}(X)$ ( $T$ exists by Lemma 2 ). Define the new directed graph $E_{X}$ which is called the $X$-corner of $E$, as follows:

$$
\begin{gathered}
E_{X}^{0}:=\left\{Q_{u}: u \in V(T)\right\}, \\
E_{X}^{1}:=\left\{T_{e, u}: u \in V(T)\right\}, \\
s\left(T_{e, u}\right):=Q_{u}, \\
r\left(T_{e, u}\right):=Q_{s(e)}
\end{gathered}
$$

Now Proposition 5 gives a $K$-homomorphism $\pi: L_{K}\left(E_{X}\right) \rightarrow L_{K}(E)$ which maps each vertex $Q_{u} \in E_{X}^{0}$ and each edge $T_{e, u} \in E_{X}^{1}$ of $L_{K}\left(E_{X}\right)$ to $Q_{u}$ and $T_{e, u}$ in $L_{K}(E)$ respectively.

In the following, we will prove that $\pi$ is injective and its image is $P_{X} L_{K}(E) P_{X}$, where

$$
P_{X}=\sum_{v \in X} v
$$

Proposition 7. The map $\pi$ is injective.

Proof. Since $\operatorname{deg}\left(\pi\left(Q_{u}\right)\right)=0$ and

$$
\operatorname{deg}\left(\pi\left(T_{e, u}\right)\right)=1 \text { for all } Q_{u} \in E_{X}^{0}, T_{e, u} \in E_{X}^{1},
$$

it is easy to see that $\pi$ is a graded ring homomorphism. Moreover, $\pi\left(Q_{u}\right) \neq 0$ for all $Q_{u} \in E_{X}^{0}$, and in view of the Graded Uniqueness Theorem [5, Theorem 4.8] it follows that $\pi$ is injective.

Proposition 8. $\pi\left(L_{K}\left(E_{X}\right)\right)=P_{X} L_{K}(E) P_{X}$.

Proof. For every $v \in V(T)$ and $e_{u} \in E_{X}^{1}$, we have

$$
P_{X} Q_{v} P_{X}=s\left(\tau_{v}\right) \cdot Q_{v} \cdot s\left(\tau_{v}\right)=Q_{v}
$$

and

$$
P_{X} T_{e, u} P_{X}=s\left(\tau_{s(e)}\right) T_{e, u} s\left(\tau_{u}\right)=T_{e, u} .
$$

It implies that

$$
\pi\left(L_{K}\left(E_{X}\right)\right) \subseteq P_{X} L_{K}(E) P_{X} .
$$

Now we show

$$
\pi\left(L_{K}\left(E_{X}\right)\right) \supseteq P_{X} L_{K}(E) P_{X} .
$$

To do this, we will show that the range of $\pi$ contains all products $\mu v^{*}$ such that

$$
\mu, v \in E^{*} ; s(\mu), s(v) \in X ;
$$

and

$$
r(\mu)=r(v) \text {. }
$$

Observe that for such $\mu$ and $v$, one has

$$
\mu v^{*}=\mu \tau_{r(\mu)}^{*} \tau_{r(\mu)} v^{*}=\left(\mu \tau_{r(\mu)}^{*}\right)\left(v \tau_{r(v)}^{*}\right)^{*},
$$

so we may assume that $v=\tau_{r(\mu)}$. We shall prove this statement by induction on the length of $\mu$.

Assume that $|\mu|=0$, that is, $\mu=s(\mu) \in X$. Then

$$
\mu=\tau_{r(\mu)} \text { and } \mu \tau_{r(\mu)}^{*}=\tau_{r(\mu)} \tau_{r(\mu)}^{*},
$$

which is in the range of $\pi$ by Lemma 3 . Now for $n \in \mathbb{N}$, assume that $|\mu|=n$ and $v \tau_{r(v)}^{*}$ is in the range of $\pi$ for every path $v$ of length $n-1$. Let $e$ be the final edge of $\mu$, and write $\mu=\mu^{\prime} e$. Then

$$
\begin{aligned}
\mu \tau_{r(\mu)}^{*} & =\mu^{\prime} \cdot e \cdot \tau_{r(e)}^{*} \\
& =\mu^{\prime} \cdot r\left(\mu^{\prime}\right) \cdot e \cdot \tau_{r(e)}^{*} \\
& =\mu^{\prime} \tau_{r\left(\mu^{\prime}\right)}^{*} \tau_{r\left(\mu^{\prime}\right)} \cdot e \cdot \tau_{r(\mu)}^{*} \\
& =\mu^{\prime} \tau_{r\left(\mu^{\prime}\right)}^{*}\left(\tau_{r\left(\mu^{\prime}\right)} \cdot e \cdot \tau_{r(e)}^{*}\right),
\end{aligned}
$$

where $\mu^{\prime} \tau_{r\left(\mu^{\prime}\right)}^{*}$ is in the range of $\pi$ by the inductive hypothesis.

If $e \in T^{1}$ then $\tau_{r\left(\mu^{\prime}\right)} e=\tau_{r(e)}$, and, hence, $\tau_{r\left(\mu^{\prime}\right)} e \tau_{r(e)}^{*}$ is in the range of $\pi$ by Lemma 3. If $e$ does not belong to $T^{1}$, then once again we use Lemma 3 to give

$$
\begin{aligned}
\tau_{r\left(\mu^{\prime}\right)} e \tau_{r(e)}^{*} & =\tau_{r\left(\mu^{\prime}\right)} e \tau_{r(e)}^{*}\left(\tau_{r(e)} \tau_{r(e)}^{*}\right) \\
& =\tau_{s(e)} e \tau_{r(e)}^{*}\left(\sum_{u \in V(T), r(e) \geq_{T} u} Q_{u}\right) \\
& =\sum_{u \in V(T), r(e) \geq_{T} u} T_{e, u} .
\end{aligned}
$$

which is in the range of $\pi$. By induction, the proof 
is completed.

Theorem 9 (Main Theorem). Let $E$ be a directed graph, $K$ a field and $L_{K}(E)$ the Leavitt path algebra of $E$ over $K$. Assume that $X$ is a subset of vertices in $E$ and $T$ is a row-finite, path-finite directed forest in $E$ such that $T^{r}=X$ and $T^{0}=$ $H_{E}(X)$. If $P_{X}=\sum_{v \in X} v$, then there exists a graph $E_{X}$ such that the corner $P_{X} L_{K}(E) P_{X}$ is isomorphic to the Leavitt path algebra $L_{K}\left(E_{X}\right)$ of $E_{X}$.

Proof. The result follows from Definition 6, Propositions 7 and 8.

\section{SOME EXAMPLES}

Example 1. Let $E$ be the graph

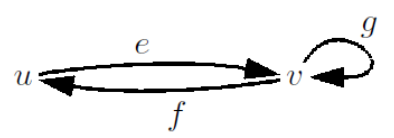

a) Let $X=\{u\}, T^{0}=E^{0}, T^{1}=\{e\}$. We have

$$
\begin{aligned}
& V(T)=\{v\}, \\
& E_{X}^{0}=\left\{Q_{v}=e e^{*}\right\}, \\
& E_{X}^{1}=\left\{T_{f, v}=e f e e^{*}, T_{g, v}=e g e^{*}\right\} .
\end{aligned}
$$

Then the corner $u L_{K}(E) u$ is isomorphic to the Leavitt path algebra of the following graph:

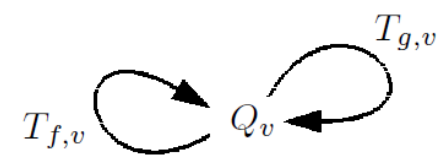

b) Let $X=\{v\}, T^{0}=E^{0}, T^{1}=\{f\}$. We obtain

$$
\begin{aligned}
& V(T)=\{u, v\}, \\
& E_{X}^{0}=\left\{Q_{u}=f f^{*}, Q_{v}=g g^{*}\right\}, \\
& E_{X}^{1}=\left\{T_{e, v}=\text { fegg }^{*}, T_{g, v}=g g g^{*},\right. \\
& \left.\qquad T_{e, u}=\text { feff }^{*}, T_{g, u}=g f f^{*}\right\} .
\end{aligned}
$$

Then the corner $v L_{K}(E) v$ is isomorphic to the
Leavitt path algebra of the following graph:

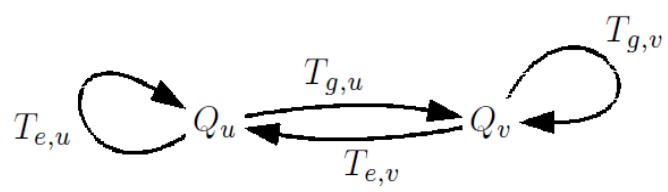

Example 2. Let $E$ be the graph

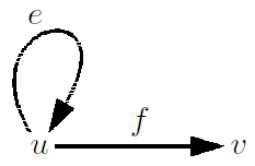

Let $X=\{u\}, T^{0}=E^{0}, T^{1}=\{f\}$. We obtain

$$
\begin{aligned}
& V(T)=\{u, v\}, \\
& E_{X}^{0}=\left\{Q_{u}=e e^{*}, Q_{v}=f f^{*}\right\}, \\
& E_{X}^{1}=\left\{T_{e, u}=e e e^{*}, T_{e, v}=e f f^{*}\right\} .
\end{aligned}
$$

Then the corner $u L_{K}(E) u$ is isomorphic to the Leavitt path algebra of the following graph:

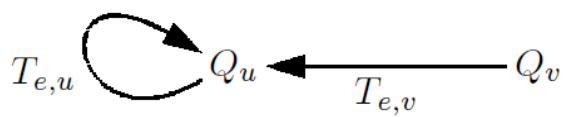

Acknowledgments: This research is funded by Vietnam National University Ho Chi Minh City (VNU-HCM) under grant number B2016-18-01.

\section{REFERENCES}

[1]. G. Abrams, G. Aranda-Pino, Purely infinite simple Leavitt path algebras, J. Pure Appl. Algebra, 207, 553$563,2006$.

[2]. G. Abrams, G. Aranda Pino, The Leavitt path algebra of a graph, J. Algebra 293, 319-334, 2005.

[3]. P. Ara, M.A. Moreno, E. Pardo, Nonstable $K$-theory for graph algebras, Alg. Represent. Theory 10, 157-178, 2007.

[4]. T. Crisp, Corners of Graph Algebras, J. Operator Theory, 60 101-119, 2008.

[5]. M. Tomforde, Uniqueness theorems and ideal structure for Leavitt path algebras, J. Algebra, 318, 270-299, 2007 


\title{
Lớp các góc của đại số đường đi Leavitt
}

\author{
Trịnh Thanh Đèo \\ Trường Đại học Khoa học Tự nhiên, ĐHQG-HCM \\ Corresponding author: ttdeo@hcmus.edu.vn
}

Ngày nhận bản thảo: 03-01-2018, Ngày chấp nhận đăng: 07-03-2018, Ngày đăng:15-10-2018.

\begin{abstract}
Cho $E$ là một đồ thị có hướng, $K$ là trường và $L_{K}(E)$ là đại số đường đi Leavitt của $E$ trên $K$. Mục tiêu của bài báo này là mô tả cấu trúc của một lớp các góc của đại số đường đi Leavitt $L_{K}(E)$. Động lực của việc nghiên cứu này đến từ bài báo "Corners of Graph Algebras" của Tyrone Crisp, trong đó góc của đồ thị $C^{*}$-đại số đã được mô tả hoàn toàn. Sử dụng cùng ý tưởng với Tyrone
\end{abstract}

Crisp, chúng tôi chỉ ra rằng với mọi con hữu hạn $X$ của tập đỉnh trong đồ thị $E$ sao cho tập họ̣p con di truyền $H_{E}(X)$ sinh bởi $X$ là hũu hạn, vành góc $\left(\sum_{v \in X} v\right) L_{K}(E)\left(\sum_{v \in X} v\right)$ của $L_{K}(E)$ đẳng cấu với với đại số đường đi Leavitt $L_{K}\left(E_{X}\right)$ của một đồ thị $E_{X}$ nào đó. Chúng tôi cũng cung cấp một cách thức để xây dựng đồ thị $E_{X}$ này.

Index Terms-Đại số đường đi Leavitt, đồ thị, góc. 